\title{
Epidemiological Analysis of Avulsion Fractures in Dogs
}

\author{
Alyssa M. Matos Cruz ${ }^{1}$ Mitsunori Kayano ${ }^{2}$ Mizuki Tomihari ${ }^{2}$ Po-Yen Chou ${ }^{3 \odot}$ Sun Young $\mathrm{Kim}^{4 \odot}$ \\ Amy S. Kapatkin ${ }^{3}$ Kei Hayashi ${ }^{1}$
}

1 Department of Clinical Sciences, College of Veterinary Medicine, Cornell University, Ithaca, New York, United States

2 Department of Veterinary Medicine, Research Center for Global Agromedicine, Obihiro University of Agriculture and Veterinary Medicine, Obihiro, Japan

${ }^{3}$ Department of Surgical and Radiological Sciences, Department of Surgical \& Radiological Sciences, College of Veterinary Medicine, University of California - Davis, Davis, California, United States

${ }^{4}$ Department of Small Animal Clinical Sciences, College of Veterinary

Medicine, Michigan State University, East Lansing, Michigan, United States

\author{
Address for correspondence Alyssa M. Matos Cruz, DVM, Department \\ of Clinical Sciences, College of Veterinary Medicine, Cornell \\ University, Ithaca, NY 14853, United States \\ (e-mail: alyssamatos24@gmail.com).
}

VCOT Open 2020;3:e60-e65.

\begin{abstract}
Keywords

- avulsion

- fracture

- orthopaedic

- epidemiology

- dog

Objective This study aimed to analyse the epidemiological data of canine appendicular avulsion fractures from three academic veterinary hospitals in the United States. Study Design A total of 114 avulsion fractures that presented to three study centres (teaching hospitals in New York, California, and Michigan) between 2008 and 2018 were analysed for avulsion type, breed, sex, body weight, age and affected side.

Results The mean age for avulsion fractures was 1.6 years ( \pm 2.3 standard deviation), and side and sex were comparable (53\% females and $54 \%$ left sided, only $2 \%$ bilateral). Tibial tuberosity fracture was the most common type, comprising $54 \%$ of all avulsion fractures, significantly more prevalent in younger and lighter dogs $(p<0.001)$. There was no significant difference in avulsion type, breed, sex, weight, age and affected side among three study centres $(p=0.66)$.

Conclusion The most common avulsion fractures were tibial tuberosity fractures, which tend to occur in young, small dogs. There is no sex or side predilection for avulsion fractures noted in this study, although the effect of neuter could not be determined. There were no differences in fracture characteristics among the three different geographical locations in the United States.
\end{abstract}

\section{Introduction}

An avulsion fracture occurs when a bone fragment detaches from the main part of bone at the attachment site of tendon, ligament or joint capsule as a result of physical trauma. Tensile forces often cause an avulsion fracture, and shear forces also play an important role in its mechanism of injury. An avulsion fracture is considered uncommon compared with all other types of fractures; however, when they occur, they still cause severe pain and functional disability in dogs. An avulsion fracture commonly occurs near the joints and may involve the physis in growing animals, which generally

received

December 14, 2019

accepted

April 29, 2020
DOI https://doi.org/

10.1055/s-0040-1713123. ISSN $2625-2325$. requires immediate surgical intervention..$^{1-5}$ Despite its clinical significance, little is known about the epidemiological aspects of avulsion fractures in dogs.

Current textbooks describe avulsion fractures of the following anatomical locations, including the acromion process (origin of the acromial part of deltoid muscle), supraglenoid tubercle (origin of biceps brachii muscle), greater tubercle (insertion of supraspinatus muscle), olecranon (insertion of triceps brachii muscle), ulnar styloid (lateral collateral ligament), radial styloid (medial collateral ligament), accessory carpal bone (carpal ligaments and insertion of flexor carpi ulnaris), ischial tuberosity (origins of biceps femoris,
License terms Stuttgart · New York

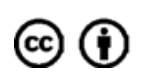


semitendinosus and semimembranosus muscles), femoral head (round ligament of the femur), greater trochanter (insertions of gluteal muscles), femoral origin of gastrocnemius muscle, femoral and tibial insertions of cranial cruciate ligament, femoral insertion of medial collateral ligament, femoral origin of long digital extensor muscle, tibial tuberosity (quadriceps femoris), medial malleolus (medial collateral ligament), lateral malleolus (lateral collateral ligament) and calcaneus (insertion of gastrocnemius muscle). ${ }^{3-5}$ The diagnosis and treatment outcomes of avulsion fractures have been sparsely reported in veterinary literature and most of them are single case reports. At present, clinical characteristics and rate of incidence of avulsion fracture in dogs are not well documented.

The goal of this study is to analyse the epidemiological data of canine appendicular avulsion fractures from three large referral centres in the United States. Epidemiological studies may provide insight to understand the manifestation of an injury and to help construct a practical differential list for dogs with a history of trauma and lameness in daily practice. In this study, we analysed data on avulsion fracture type, breed, sex, weight, age and affected side from three academic veterinary hospitals across the United States (East Coast, Midwest and West Coast).

\section{Materials and Methods}

A keyword search with avulsion fracture was conducted on available medical database at Cornell University College of Veterinary Medicine (Cornell; Universal Veterinary Information System), University of California Davis (UCD; Veterinary Hospital Information System), and Michigan State University (MSU; Vetstar) for the period of 2008 to 2018. Inclusion criteria were canine patients of all breeds, who were diagnosed with an appendicular avulsion fracture by imaging (radiographs, computed tomography, magnetic resonance imaging) interpreted at the time by a board-certified radiologist or by a board-certified surgeon. Breed, sex, body weight, age, side of the lesion and type of fractures were recorded. Avulsion fractures in the carpal area were grouped into one 'carpal' avulsion fracture category. Avulsion fractures in the tarsus were grouped into one 'tarsal' avulsion fracture category, except for malleolar fractures which were grouped into a separate group of 'malleolus' avulsion fracture category. Patients whose records and/or imaging were insufficient were excluded from the study as well as dogs that underwent surgery prior to fracture. Dogs that had a cranial cruciate ligament avulsion fracture were excluded, as the definitive diagnosis was not obtained in many cases. Neuter status could not be determined due to the inconsistency of recording style in the database.

The difference of types of avulsion fracture, breed, sex, injured side, age and weight, among three study centres (CU, UCD, MSU) was examined by chi-squared tests. The difference of age and weight among the study centres was compared by analysis of variance. Kruskal-Wallis test and SteelDwass test were applied for the difference of age and weight between six common avulsion types. Spearman's rank cor- relation coefficient was calculated to analyse the correlation of age and weight. All statistical analyses were performed by the use of statistical software (RStudio, version 1.1.463 for Mac, Boston, Massachusetts, United States). The significance level was set to be 0.05 .

\section{Results}

A total of 114 avulsion fractures met the inclusion criteria (-Table $\mathbf{1}$ ). The overall mean age was 1.6 years old $( \pm 2.3$ standard deviation) with an overall mean weight of $18.48 \mathrm{~kg}$ ( \pm 11.48 standard deviation). No significant difference was observed in type of avulsion fracture, breed, sex, injured side, age or body weight among the three study centres. Tibial tuberosity fractures were the most common avulsion fracture at every study centre, overall comprising $54 \%$ of all avulsion fractures (-Fig. 1). The most common types of avulsion fractures were in order of frequency: tibial tuberosity (62/114), malleolar (10/114), carpal (6/114), supraglenoid tubercle (5/114) and greater trochanter (4/114). Breeds most commonly affected were mixed breed (26/114), Labrador Retriever (15/114), American Pit Bull Terrier (5/114), Australian Shepherd (5/114), French Bulldog (5/114) and Rottweiler (5/114). Among the 114 dogs, 60 were female (53\%) and 54 were male (47\%). Sixty-one cases had left-sided fractures (53\%), and fifty-one had right-sided fractures (45\%) and two were affected bilaterally ( $2 \%$ ).

The distribution of age and weight was significantly different between the types of avulsion fractures $(p<0.001$; - Figs. 2 and 3). Carpal avulsion fractures tended to be observed in older dogs $(p<0.10)$. Tibial tuberosity fractures occurred in younger dogs $(p<0.001)$. Tibial tuberosity fractures were also observed in lighter dogs, but a confounding effect due to age needs to be considered. In fact, age and weight were significantly correlated with each other $(r=0.424, p<0.001)$. Therefore, the true factor, which can be different in fracture type, might be age.

\section{Discussion}

There are no recent comprehensive studies analysing avulsion fracture in dogs. This study evaluated the epidemiological characteristics of all types of appendicular avulsion fractures in three regions of the United States, by analysing cases seen at three academic institutions in the past 10 years. Avulsion fractures appeared to be much less common compared with other types of fractures at these study centres ( $<5 \%$ of all fractures, unpublished data). The most commonly diagnosed avulsion fracture was tibial tuberosity fracture.

The results found in this study regarding tibial tuberosity fractures are similar to those in previous studies where most of these fractures are reported in young animals. There have been several studies analysing the pathology of tibial tuberosity avulsion fractures specifically. In a study by Skelly et al, tibial tuberosity avulsion fractures in a litter of greyhound puppies were found in six out of seven puppies, some bilaterally. The study noted histopathological changes suggestive of osteochondritis. ${ }^{6}$ Another study by Gower et al 
Table 1 Summary of sample characteristics of avulsion fractures at three study centres

\begin{tabular}{|c|c|c|c|c|}
\hline \multicolumn{5}{|c|}{ Avulsion summary } \\
\hline & \multicolumn{4}{|l|}{ Study centre } \\
\hline & All & Cornell & UCD & MSU \\
\hline Number & 114 & 31 & 30 & 53 \\
\hline Gender (M/F) & $54 / 60$ & $11 / 20$ & $13 / 17$ & $30 / 23$ \\
\hline Age $(y \pm S D)$ & $1.60 \pm 2.30$ & $1.72 \pm 2.42$ & $1.85 \pm 2.48$ & $1.39 \pm 2.15$ \\
\hline Weight $(\mathrm{kg} \pm \mathrm{SD})$ & $18.48 \pm 11.48$ & $18.55 \pm 11.82$ & $21.68 \pm 11.20$ & $16.64 \pm 11.25$ \\
\hline Side (L/R/Bilat) & $61 / 51 / 2$ & $16 / 15 / 0$ & $17 / 13 / 0$ & $28 / 23 / 2$ \\
\hline \multirow[t]{7}{*}{ Type } & Tibial tuberosity 62 & Tibial tuberosity 15 & Tibial tuberosity 16 & Tibial tuberosity 31 \\
\hline & Malleolus 10 & Malleolus 4 & Malleolus 3 & Malleolus 3 \\
\hline & Carpal 6 & Supraglenoid tubercle 2 & Carpal 3 & Carpal 2 \\
\hline & Supraglenoid tubercle 5 & Carpal 1 & Supraglenoid tubercle 1 & Supraglenoid tubercle 2 \\
\hline & Greater trochanter 4 & Greater trochanter 1 & Greater trochanter 1 & Greater trochanter 2 \\
\hline & Others 27 & Others 8 & Others 6 & Tarsal 2 \\
\hline & & & & Others 11 \\
\hline \multirow[t]{8}{*}{ Breed } & $\operatorname{Mix} 26$ & Mix 7 & Mix 7 & $\operatorname{Mix} 12$ \\
\hline & Labrador Retriever 15 & Labrador Retriever 7 & Labrador Retriever 4 & Labrador Retriever 4 \\
\hline & PitBull Terrier 5 & German Shepherd 2 & PitBull Terrier 3 & French Bulldog 4 \\
\hline & Australian Shepherd 5 & Australian Shepherd 1 & Rottweiler 2 & Australian Sheperd 3 \\
\hline & French Bulldog 5 & Rottweiler 1 & Australian Shepherd 1 & PitBull Terrier 2 \\
\hline & Rottweiler 5 & PitBull Terrier 0 & French Bulldog 1 & Rottweiler 2 \\
\hline & Others 53 & French Bulldog 0 & Others 12 & Others 26 \\
\hline & & Others 13 & & \\
\hline
\end{tabular}

Abbreviations: MSU, Michigan State University; SD, standard deviation; UCD, University of California, Davis.

revealed that $86 \%$ of affected animals $(51 / 59)$ were Staffordshire Bull Terriers with a mean age of 5 months. ${ }^{7}$ These studies (from Ireland and the United Kingdom) allude at higher incidence on certain breeds, whereas our study from three remote locations in the United States suggested that there was no breed predominance in the United States. The previous results might be skewed based on region and general breed predominance on a particular area.

Interestingly, recent studies reported atypical clinical presentation of tibial tuberosity fractures, proposing atraumatic pathology. ${ }^{8,9}$ Namely, von Pfeil et al study discusses the relationship between predisposing diseases (such as osteochondritis and Osgood-Schlatter disease) and tibial tuberosity avulsion in dogs. ${ }^{8}$ However, our study did not evaluate aetiology of avulsion fractures. Particularly for tibial tuberosity avulsion fractures, assessment of traumatic versus atraumatic events would add substantial value, and future studies should focus on aetiology.

Beside tibial tuberosity fractures, other less common avulsion fractures have been only sparsely reported in dogs. Previous studies described radiographic appearance of intra-articular stifle avulsion fractures (cranial cruciate ligament and long digital extensor tendon). ${ }^{10,11}$ More recent case studies described diagnostic procedures and treatment outcome of avulsion fractures near the shoulder joint (infraspinatus and supraspinatus) ${ }^{12}$ and supraglenoid tuberosity, ${ }^{13}$ elbow joint (biceps and triceps) ${ }^{14-16}$ and hip joint (lesser trochanter). ${ }^{17}$ Many of the reports are single cases in the format of "What is Your Diagnosis" in the Journal of American Veterinary Medical Association. These case reports include avulsion fractures of the caudal cruciate ligament, origins of gastrocnemius and popliteal muscles and various ligaments in carpal and tarsal regions. ${ }^{18-23}$

Biomechanical factors related to avulsion fractures in dogs have so far not been discussed in different facture locations. Although tensile forces produced by muscle contraction is classically attributed to cause avulsion fractures, shear forces also play an important role in their mechanism of injury. ${ }^{3-5}$ As shown in this study, malleolar and carpal avulsion fractures were the second and third most common avulsion fractures in dogs in the United States. These types of fractures are considered to occur from trauma; hence, shear forces must play an important role in its injury mechanism. The tibial tuberosity serves as the insertion site of the quadriceps femoris muscle, and avulsion of the tuberosity can result from contraction of the muscle, while the stifle joint is flexed and the foot is set firmly on the ground. ${ }^{5}$ 

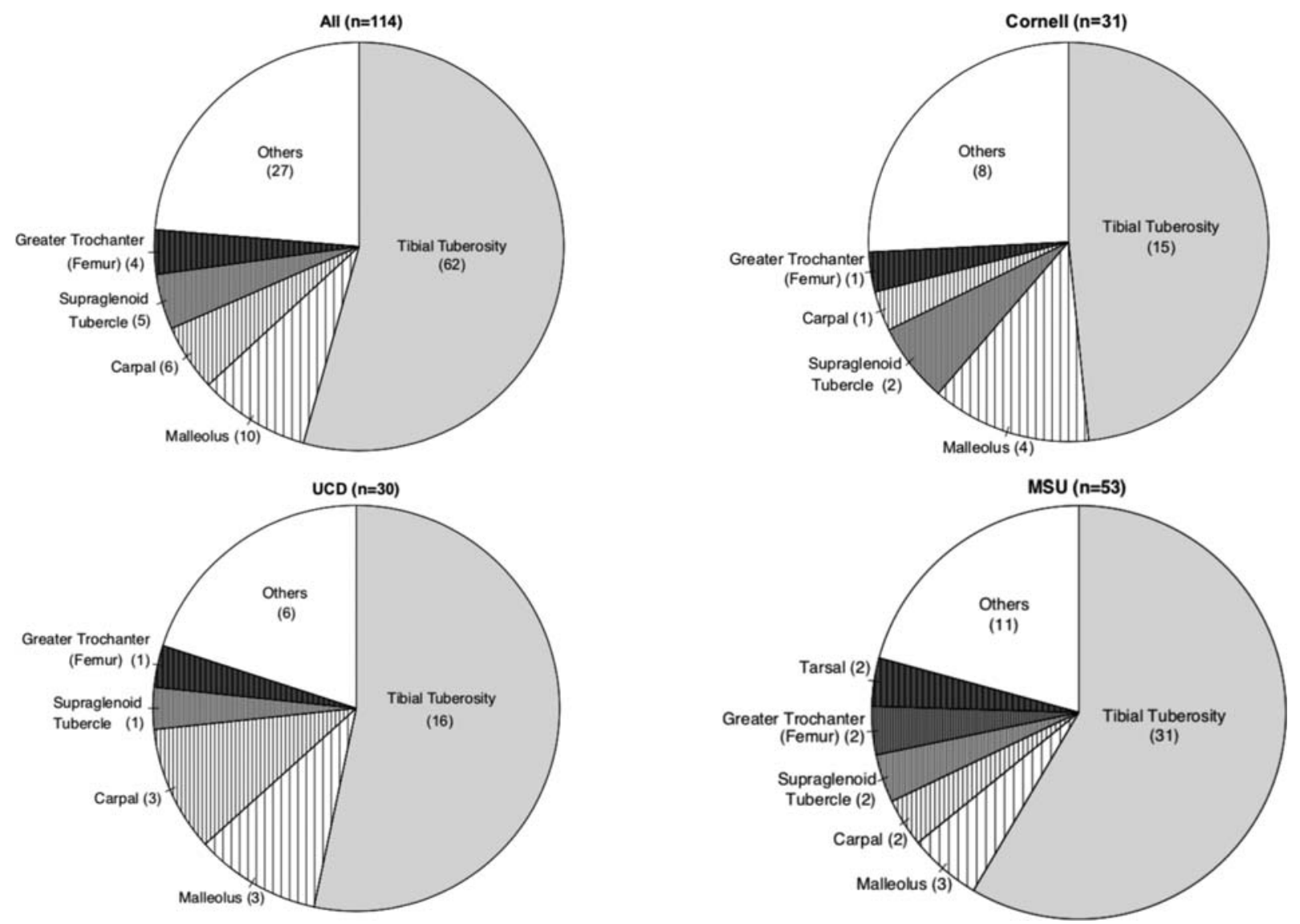

Fig. 1 Frequency of occurrence of different types of avulsion fracture at referral centres. MSU, Michigan State University; UCD, University of California, Davis.

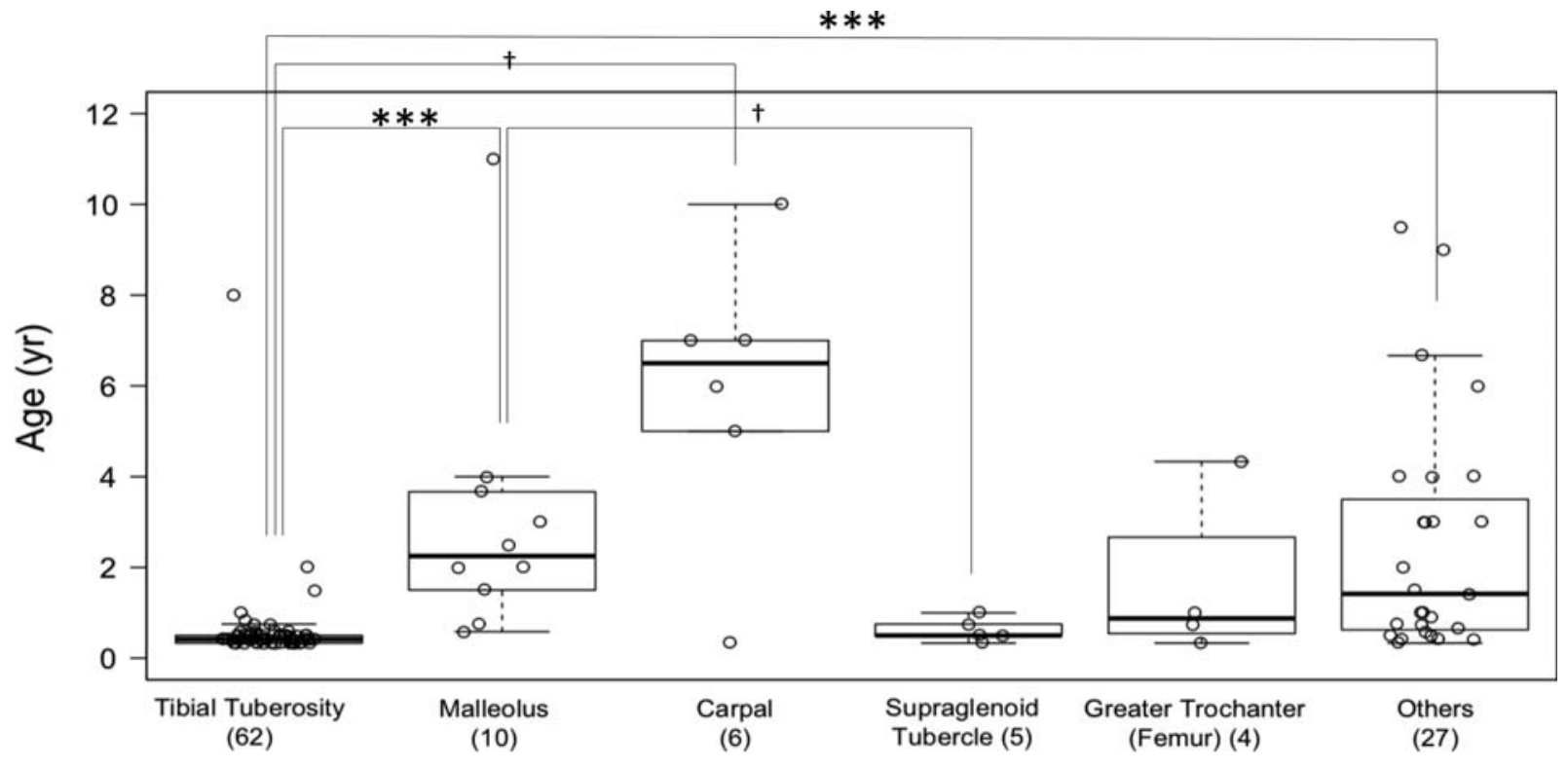

Fig. 2 Box plot of the effect of age on types of avulsion fracture. Strong significance $(p<0.001)$ and tendency $(p<0.10)$ are shown by ${ }^{* * *}$ and $\dagger$, respectively. Heavy lines represent medians, boxes are the range from the first quantile $\left(Q_{1}\right)$ to the third quantile $\left(Q_{3}\right)$ and whiskers show the maximum and minimum data points in $\pm 1.5\left(Q_{3}-Q_{1}\right)$ from the edges of the box.

Fracture of the medial or lateral malleolus often occurs with shearing injury but may occur as an isolated fracture. ${ }^{5}$

Analysis of aetiology, as well as other confounding factors such as body condition score, obesity, activity level, possibly diet, rate of growth, lifestyle (i.e. working dog versus solely companion animal), region (countryside versus urban area), injury mechanisms and type of trauma (atraumatic incident versus low energy versus high energy trauma) would provide with pertinent information that may help decreasing the incidence of this type of injury in the future. 


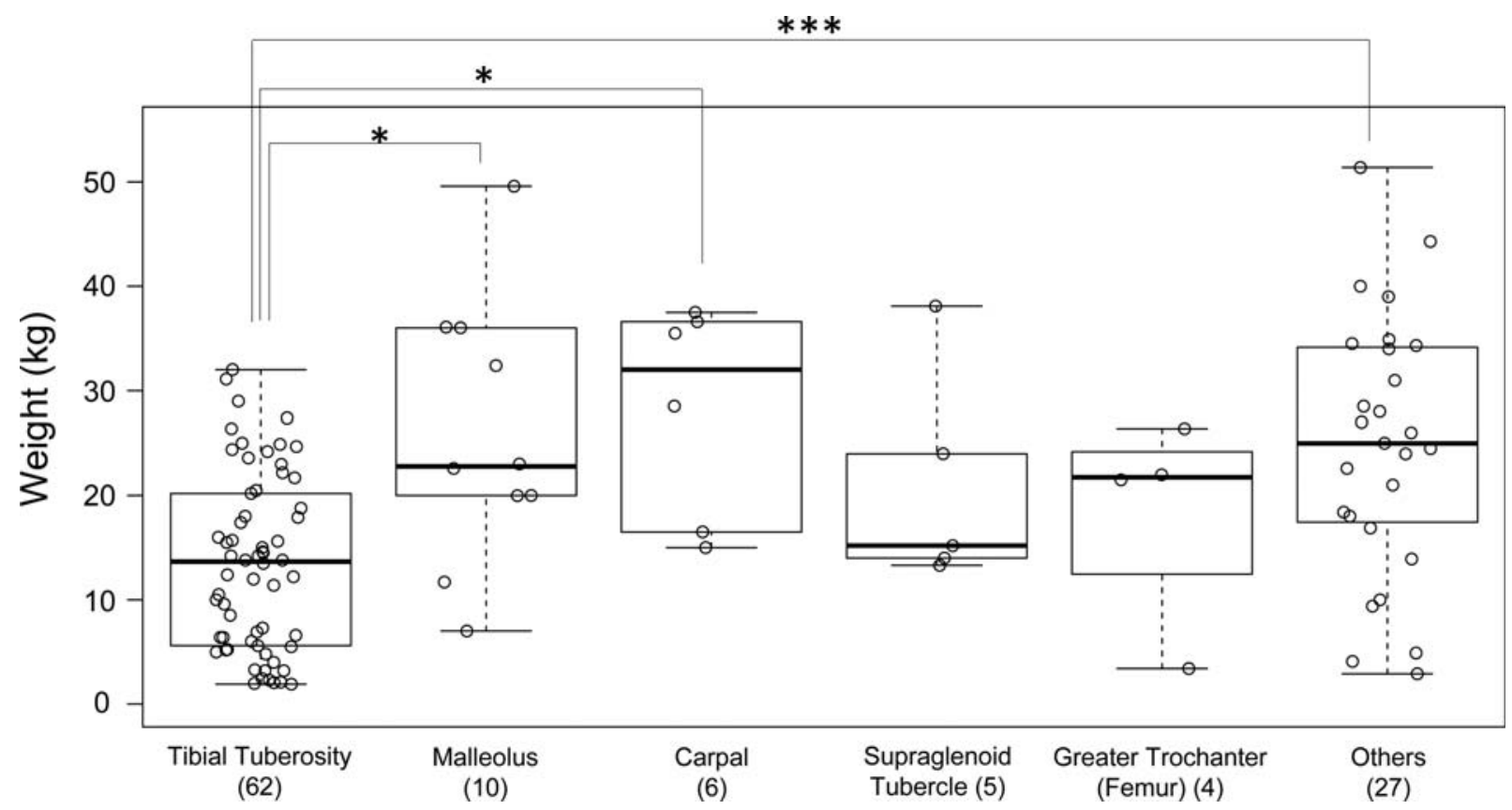

Fig. 3 The effect of body weight on types of avulsion fracture. Strong $(p<0.001)$ and standard significance $(p<0.05)$ are shown by ${ }^{* * *}$ and *, respectively. Heavy lines represent medians, boxes are the range from the first quantile $\left(Q_{1}\right)$ to the third quantile $\left(Q_{3}\right)$, and whiskers show the maximum and minimum data points in $\pm 1.5\left(Q_{3}-Q_{1}\right)$ from the edges of the box.

In this study, there is a relationship between age and body weight, but they were significantly correlated with each other and a true factor was not clearly identified. In addition, correlating body weight to standard body weights to evaluate role of obesity with avulsion fractures was not possible, given we did not collect data on body condition scores nor the numbers of one specific breed were sufficient to make the analysis significant.

Thus, a limitation of the study is the lack of information on aetiology of avulsion fractures, as discussed above. Another limitation is the uncertainty of true incidence of avulsion fractures in comparison to other orthopaedic conditions in dogs. For example, odds ratio statistics could have enhanced this study. We also did not evaluate the effect of neutering on the incidence of fractures, since our study did not evaluate factors related to aetiology; further investigation might be beneficial to determine a way to prevent its occurrence. Hereditary and developmental factors might be associated with these types of injury of young dogs, especially with tibial tuberosity avulsion fractures. Our study is comprised of only small numbers of other avulsion fracture types; hence, our results regarding other fractures might not be statistically meaningful due to the limited sample size. Other specific types of avulsion fractures might warrant further investigation. Notably, taking in consideration our finding that carpal fractures tend to occur in older animals, it might indicate that not all avulsion fractures have same or similar aetiologies.

\section{Conclusion}

The most common avulsion fractures were tibial tuberosity fractures in young and lighter dogs. There were no other clinically relevant trends in avulsion fracture type based on geographical location, breed, sex, body weight and affected side.

\section{Funding}

None.

Conflict of Interest

None declared.

\section{References}

1 Johnson JA, Austin C, Breur GJ. Incidence of canine appendicular musculoskeletal disorders in 16 veterinary teaching hospitals from 1980 through 1989. Vet Comp Orthop Traumatol 1994;07 (02):56-69

2 Unger M, Montavon PM, Heim UFA. Classification of fractures of long bones in the dog and cat: introduction and clinical application. Vet Comp Orthop Traumatol 1990;03(02):41-50

3 DeCamp CE, Johnston SA, Déjardin Loïc M, et al. Brinker, Piermattei and Flos Handbook of Small Animal Orthopedics and Fracture Repair. Fifth edition. St. Louis, MO: Elsevier; 2016

4 Fossum TW. Small Animal Surgery. Fifth edition. Philadelphia, PA: Elsevier; 2019

5 Johnston SA, Tobias KM. Chapters 61, 63, 64, 70. Veterinary Surgery: Small Animal. Second edition. St. Louis, MO: Elsevier; 2018

6 Skelly CM, McAllister H, Donnelly WJ. Avulsion of the tibial tuberosity in a litter of greyhound puppies. J Small Anim Pract 1997;38(10):445-449

7 Gower JA, Bound NJ, Moores AP. Tibial tuberosity avulsion fracture in dogs: a review of 59 dogs. J Small Anim Pract 2008;49(07):340-343

8 von Pfeil DJ, Decamp CE, Ritter M, et al. Minimally displaced tibial tuberosity avulsion fracture in nine skeletally immature large breed dogs. Vet Comp Orthop Traumatol 2012;25(06):524-531

9 Brown GW, Kalff S, Parry A, Whitehead M, McKee WM. Avulsion fragmentation of the tibial tuberosity apophysis and associated patellar tendon enthesopathy in a skeletally immature dog. Vet Comp Orthop Traumatol 2013;26(03):242-247 
10 Williams J, Fitch RB, Lemarié RJ. Partial avulsion of the origin of the cranial cruciate ligament in a 4 year-old dog. Vet Radiol Ultrasound 1997;38(05):380-383

11 Fitch RB, Wilson ER, Hathcock JT, Montgomery RD. Radiographic, computed tomographic and magnetic resonance imaging evaluation of a chronic long digital extensor tendon avulsion in a dog. Vet Radiol Ultrasound 1997;38(03):177-181

12 Mikola K, Piras A, Hakala L. Isolated avulsion of the tendon of insertion of the infraspinatus and supraspinatus muscles in five juvenile Labrador Retrievers. Vet Comp Orthop Traumatol 2018; 31(04):285-290

13 Deneuche AJ, Viguier E. Reduction and stabilisation of a supraglenoid tuberosity avulsion under arthroscopic guidance in a dog. J Small Anim Pract 2002;43(07):308-311

14 Schaaf OR, Eaton-Wells R, Mitchell RAS. Biceps brachii and brachialis tendon of insertion injuries in eleven racing greyhounds. Vet Surg 2009;38(07):825-833

15 Ambrosius L, Arnoldy C, Waller KR III, Little JP, Bleedorn JA. Reconstruction of chronic triceps tendon avulsion using synthetic mesh graft in a dog. Vet Comp Orthop Traumatol 2015;28(03): 220-224

16 García-Fernández P, Quero Martín P, Mayenco A, Gardoqui M, Calvo I. Surgical management and follow-up of triceps tendon avulsion after repeated local infiltration of steroids: two cases. Vet Comp Orthop Traumatol 2014;27(05):405-410
17 Vidoni B, Henninger W, Lorinson D, Mayrhofer E. Traumatic avulsion fracture of the lesser trochanter in a dog. Vet Comp Orthop Traumatol 2005;18(02):105-109

18 Monotti IC, Davies SE, Lidbetter DA. What is your diagnosis? Caudal cruciate avulsion fracture. J Am Vet Med Assoc 2015; 246(05):507-509

19 Horowitz FB, Wells KL. What is your diagnosis? Dorsomedial avulsion of the right second carpal bone with medial displacement of the proximal portion of the second metacarpal bone. J Am Vet Med Assoc 2007;230(01):31-32

20 Nanfelt M, Zekas LJ, Palmer RH. What is your diagnosis?: Oblique fracture of the talus with subluxation of the talocalcaneal joint and avulsion fracture of the lateral malleolus J Am Vet Med Assoc 2006;229(03):357-358

21 Ting D, Petersen SW, Mazzaferro EM, Worth LT, Petersen SW; American College of Veterinary Radiology. What is your diagnosis? Avulsion of the origin of the gastrocnemius muscle. J Am Vet Med Assoc 2006;228(10):1497-1498

22 Bleedorn JA, Towle HA, Breur GJ, Rohleder JJ. What is your diagnosis? Avulsion of the popliteal tendon. J Am Vet Med Assoc 2006;229(12):1885-1886

23 Vinayak A, Weigel JP, Henry GA, Johnson CA. What is your diagnosis? Avulsion fracture of the plantar aspect of the distal end of the calcaneus and plantar proximal intertarsal subluxation. J Am Vet Med Assoc 2004;224(01):39-40 